\title{
Marketing científico digital e métricas alternativas para periódicos: da visibilidade ao engajamento
}

Ronaldo Ferreira de Araújo

\author{
Professor do Curso de Biblioteconomia, \\ Universidade Federal de Alagoas \\ (UFAL).Doutorando em Ciência da Informação, \\ Universidade Federal de Minas Gerais (UFMG)
}

http://dx.doi.org/10.1590/1981-5344/2402

O artigo reflete sobre o papel do marketing científico digital no aumento da visibilidade dos periódicos científicos bem como do acompanhamento e avaliação do seu impacto social por meio da altmetria. Para tanto apresenta esse tipo de marketing como forma de promoção e divulgação dos periódicos para os quais seus editores devem: construir e manter uma presença online; oferecer um conteúdo adequado aos ambientes que atuar, e; estabelecer uma atuação responsiva. Discorre sobre os principais indicadores do marketing científico digital e por fim, situa a altmetria como método para coleta e análise de tais indicadores com atenção ao custo e padrões exigidos pelos periódicos.

Palavras-chave: Marketing científico digital; Altmetria; Visibilidade de periódicos; Comunicação científica.

\section{Scientific digital marketing and altmetrics for academic journals: from visibility to engagement}

The paper reflects about the scientific digital marketing role to raise the visibility of scholarly journals as well as the monitoring and evaluation of their social impact by altmetrics. For the application of this kind of marketing the publishers should build and maintain an online presence; provide appropriate content to environments that act, and; establish a responsive performance. The main indicators of scientific digital marketing is discussed and the altmetrics is considered as a method for collecting and analyzing such indicators. Before opting for the 
altmetrics service to be used cost and the required standard of journals should be observed.

Keywords: Scientific digital marketing; Altmetrics; Journal; Visibility; Scientific communication.

Recebido em 17.04.2015 Aceito em24.07.2015.

\section{Introdução}

A audiência, o impacto social e a presença online têm sido assuntos cada vez mais debatidos quando se discute a comunicação científica no contexto do ciberespaço no qual se trabalha com a ideia de uma Ciência 2.0 (WALDROP, 2008). Esta, por sua vez, sugere novas práticas de cientistas que publicam resultados experimentais ou finais, matérias, novas teorias, reivindicações de descoberta e de projetos na web para que outros possam ver e comentar.

A ciência 2.0 pode ser percebida como aplicação das tecnologias de redes sociais nos processos científicos, sobretudo no compartilhamento de: (a) pesquisas (plataformas para publicação de conteúdos, projetos, experimentos); (b) fontes (referências, links e documentos); (c) resultados (atitude aberta de divulgação de resultados de pesquisa) (REBIUN, 2011), com potencial para beneficiar tanto as comunidades científicas quanto o público em geral.

O sucesso das tecnologias da web 2.0 como os wikis, blogs, microblogs e outras mídias sociais tem aberto novas oportunidades para colaboração e compartilhamento de informação entre cientistas (SANTOS; FEIRE; SILVA, 2009) e com isso, surgem novos indicadores que avaliam a atenção que seus trabalhos recebem e a visibilidade que atingem seus produtos de pesquisa, em especial, os artigos de periódicos.

A altmetria se apresenta como campo emergente dos estudos métricos da informação científica nesses ambientes da web 2.0 que requer mais atenção da comunidade científica. Em seu pouco tempo de chegada, pesquisadores já começam a questionar o baixo número de indicadores que as ferramentas atuais apresentam para artigos de periódicos brasileiros e da América Latina (ALPERIN, 2014; NASCIMENTO; ODDONE, 2014; ARAúJO, 2014a).

Entende-se que a altmetria serve para avaliação e mensuração da circulação da informação científica na web social e que antes de se preocupar com essa avaliação é necessário perceber dentro de que processo ela se enquadra e com que propósito. Para isso, o artigo sugere que a criação e a análise de indicadores altmétricos sejam pensadas no âmbito do marketing científico digital.

Ao refletir sobre a comunicação científica no contexto web e as implicações da comunicação eletrônica nos canais informais e formais de comunicação científica, sobretudo na (nova) visibilidade dos periódicos, abre-se caminho para apresentar o marketing científico digital como 
posicionamento coerente à aplicação da altmetria como instrumento de avaliação de novas métricas com as quais esse novo tipo de marketing lida.

\section{Visibilidade de periódicos na comunicação científica eletrônica}

O surgimento e desenvolvimento das novas tecnologias de comunicação e informação, em especial a internet, têm modificado o processo de comunicação, tanto a informal quanto a formal, estabelecendo uma nova categoria na comunicação científica: a comunicação eletrônica (OLIVEIRA; NORONHA, 2005).

As mudanças que a internet tem proporcionado à comunicação científica são substanciais, dentre elas, beneficiando-se da rede internacional de computadores, os periódicos científicos iniciaram um movimento de adoção da mídia eletrônica para publicação (BERTIN; FORTALEZA; SUHET, 2007).

A versão eletrônica, quando de acesso livre e gratuito, aumenta muito a visibilidade das revistas e a chance de ter seus artigos citados (FERREIRA; TARGINO, 2008) e seus autores mais reconhecidos.

Para Ferreira e Caregnato (2014, p. 178) "trabalhos e ideias de um pesquisador que sejam facilmente acessíveis podem ser considerados detentores de uma alta posição de visibilidade". Nessa compreensão, a visibilidade pode ser considerada "o grau de exposição e evidência de um pesquisador frente à comunidade científica" (ZIMBA; MUELLER, 2004, p. 49).

Mas torna-se necessário ressaltar que, a visibilidade do autor e não leva em consideração o reconhecimento ou a qualidade do seu trabalho, já que a exposição, por vezes, pode ser negativa (FERREIRA; CAREGNATO, 2014).

Em se tratando de periódicos científicos, um dos aspectos que compreende sua visibilidade, de uma maneira geral, é o grau de presença que apresentam nas fontes de informação da internet.

De acordo com Ferreira e Caregnato (2014, p. 178) "para ser visível, o periódico eletrônico precisa atingir membros de uma comunidade, que buscam sanar suas demandas e necessidades informacionais. Nesse caso, a visibilidade de uma publicação científica indica o seu reconhecimento".

Para os periódicos alcançarem tal prestígio e visibilidade precisam ser referência de qualidade e credibilidade em uma determinada área e estar indexados em índices de prestígio nacionais e internacionais (PACKER; MENEGHINI, 2006) preferencialmente internacionais (VILLAMÓN et al., 2009).

Segundo Ferreira e Caregnato (2014, p. 179) "as condições básicas para se obter visibilidade são a aceitação, o reconhecimento, o uso, a credibilidade e o acesso à informação". Mas... 
[...] é importante salientar que nem todas essas condições precisam estar presentes ao mesmo tempo para definir se um periódico é visível. Por esse motivo, por possuírem uma estreita ligação e dependência, em determinadas ocasiões, pode ser entendido que os itens ou os atributos que tornam a informação visível sejam definidos como visibilidade (FERREIRA; CAREGNATO, 2014, p. 179).

Packer e Meneghini (2006) apresentam alguns atributos e indicadores de visibilidade para os periódicos, dos quais, para a presente reflexão, destaca-se: (1) ser uma publicação eletrônica na internet e com acesso aberto; (2) contar com número de índices referenciais nacionais e internacionais nos quais está indexado; e (3) ter os números médios de leituras ou de artigos acessados periodicamente.

O primeiro item pode ser considerado como resolvido para a maioria das revistas brasileiras, uma vez que, a partir de 2003, as revistas impressas têm migrado e as novas têm sido criadas eletronicamente, disponíveis online e de acesso aberto, geralmente suportadas no Sistema Eletrônico de Editoração de Revistas/Open Journal Systems (SEER/OJS), plataforma gratuita de editoração eletrônica de revistas.

Para o Instituto Brasileiro de Informação em Ciência e Tecnologia (IBICT), responsável pela tradução, adaptação e distribuição do SEER, a aceitação da plataforma pela comunidade brasileira de editores científicos vem do desempenho do sistema e sua fácil adaptação aos processos de editoração em uso. O SEER apresenta qualidade de navegação na web e uma melhoria na adoção dos padrões editoriais internacionais para periódicos online 100\% eletrônicos (IBICT, 2013).

Mesmo nesse contexto e com aplicação de recursos tecnológicos no gerenciamento e editoração dos periódicos alguns autores têm percebido uma precariedade da difusão e circulação das revistas (FURNARO; RAMOS; CARVALHO, 2013) e que "algumas revistas necessitam revisar os quesitos necessários à divulgação para maior visibilidade" (CUENCA et al., 2013, p. 66).

Não se pode desconsiderar a potencialidade das ferramentas da web para a visibilidade científica. Atualmente as revistas se valem, por exemplo, da ação do e-mail marketing com envio de mensagens por meio de correio eletrônico para uma base de clientes/usuários que se cadastram para receber notificações de publicação de novas edições.

Ações de e-mail marketing tem a função de divulgar e manter contato com a base de consumidores/usuários (POTTS, 2007). A ação limita o alcance da divulgação à base de usuários cadastrados e não ultrapassa esse público, o que no contexto atual, precisa ser repensado, principalmente como combiná-lo com outras ações.

O aumento da capacidade de comunicação interativa e o crescente número de canais e fontes eletrônicas de informação faz com que seja cada vez mais necessário refletir sobre o melhor uso dessas potencialidades seja na produção, circulação, acesso e avaliação de uso 
das revistas. O marketing científico digital pode ser um aliado nessa tarefa, sobretudo para os periódicos eletrônicos.

\section{Marketing científico digital}

O marketing científico pode ser considerado, de uma maneira geral, uma modalidade de marketing que auxilia a alavancar o crescimento do mercado de produtos científicos. De acordo com Bizzocchi (2002) o princípio básico a ser adotado pelo marketing científico é o de que o conhecimento, sobretudo o discurso que o contém, é um produto como outro qualquer, bastando que se estabeleça como alvo desse produto, 0 público em geral e não somente o público especializado.

Assim, fala-se da utilização do marketing para consolidar, vender e projetar uma boa imagem da ciência e seus produtos (empresas, cientistas e instituições), e assim estimular a confiança e o investimento em pesquisas (CARVALHO, 2003).

A essência deste tipo de marketing não é a aplicação do conhecimento científico ao fazer mercadológico, mas sim o inverso disso, é a aplicação do conhecimento mercadológico ao fazer científico (BIZZOCCHI, 2002). Trata-se da própria apropriação do marketing para:

Alargar o discurso científico, a um público amplo; promover e divulgar os artigos visando o reconhecimento das pesquisas nacionais em âmbito internacional; projetar referees e autores atribuindo-Ihes prestígio e a visibilidade tão almejada (BOMFÁ et al., 2009, p.203)

Castro (2011) comenta sobre a comunicação do espanhol Félix de Moya Anegón, pesquisador do Departamento de Dinâmica da Ciência e da Inovação do Instituto de Políticas e Bens Públicos de Granada (Espanha), durante o $2^{\circ}$ Seminário de Avaliação do Desempenho dos Periódicos Brasileiros no Journal Citation Reports (JCR). O objetivo do seminário era discutir a crescente visibilidade conquistada pela ciência brasileira no JCR.

Em sua fala Moya afirma que no sistema complexo de comunicação da ciência, há capacidade para implantar o conhecimento em distintos níveis de revistas, e que nem os pesquisadores nem as revistas ficam imóveis. O que faz falta é que sejam observadas as tendências que devem seguir pesquisadores e revistas, para o pesquisador espanhol, os editores devem desenvolver atividades de marketing científico (CASTRO, 2011).

Autores como Bomfá e outros (2007) discutem o marketing aplicado a periódicos eletrônicos como 'marketing científico eletrônico', mas no contexto brasileiro, o termo marketing digital teve definitivamente maior difusão, adoção e consolidação, seja pela academia (pesquisadores e cientistas) ou pelo mercado (agências de publicidade e profissionais de marketing). 
Uma breve consulta realizada em 26 de outubro de 2014, no Google Acadêmico ${ }^{1}$, por exemplo, revela um resultado de 502 itens para busca com termo 'marketing eletrônico', contra 5.460 itens para 'marketing digital'. Sendo assim, considera-se mais adequado o emprego do conceito de 'marketing científico digital', como junção do marketing científico e marketing digital.

O marketing digital é a aplicação de estratégias no ambiente digital que utilizam teorias e ferramentas de marketing já conhecidas. Sendo assim, não é um novo marketing, e muito menos pode ser considerado uma reinvenção do marketing. Trata-se de utilizar a internet com o uma ferramenta de marketing, envolvendo comunicação, publicidade, propaganda e todo o arsenal de estratégias e conceitos já conhecidos na teoria do marketing (TORRES, 2009).

Esse tipo de marketing pode ser visto como uma resposta ao contexto atual de conectividade no qual a sociedade vive e ao novo tipo de usuário que povoa o ciberespaço. Ele atende a necessidade gerada pela inovação tecnológica da comunicação, advinda da internet, e da web 2.0 que vem alterando o comportamento do consumidor (OLIVEIRA, 2011).

Em outras palavras, pode-se dizer que o marketing digital rompe com a relação unidirecional de divulgação/disseminação do marketing convencional, uma vez que: o público alvo (usuário/cliente) também se comunica, produz conteúdo, participa - indicando um relacionamento mais estreito.

Os principais canais que têm sido explorados para esse relacionamento são os blogs e as mídias sociais como Facebook, Twitter, Instagram, dentre outras. Ambientes assim são dotados de recursos da web 2.0 de interação e colaboração. No contexto do marketing digital, seu uso, permite a criação e o compartilhamento de informações e conteúdos pelos usuários e para os usuários, nas quais o cliente é ao mesmo tempo produtor e consumidor da informação (TORRES, 2009).

Quando o marketing digital é aliado ao marketing científico considera-se o marketing científico digital como estratégia empregada em produtos da ciência, aliada à comunicação científica e comunicação digital, com o intuito de oferecer serviços alinhados às necessidades dos usuários, visando à promoção de periódicos, pesquisas e pesquisadores, com foco na visibilidade científica.

A escolha das estratégias de marketing científico digital precisa refletir o tipo de imagem que se quer transmitir, bem como atender ao perfil dos clientes/usuários que possui ou que se pretende alcançar. Vale lembrar que no ciberespaço esse público alvo ultrapassa os limites da comunidade de pesquisadores e se estende a outros segmentos da sociedade (ARAúJO; FREIRE, 1996).

Existem várias características próprias do ambiente web que podem acrescentar vantagens na aplicação do marketing científico digital, como a dinâmica e rapidez com que as mensagens se propagam, os aspectos de

\footnotetext{
${ }^{1}$ Disponível em: <http://scholar.google.com/>. Acesso em: 26 out. 2014.
} 
interatividade e a crescente articulação em rede, bem como questões de colaboração e comunicação dialógica.

Pesquisadores, editores de periódicos científicos ou instituições que pesquisa que se interessam em trilhar esse caminho e aproveitar o melhor que essas características oferecem para o emprego do marketing científico digital devem se dedicar a três questões essenciais: construir e manter uma presença online; oferecer um conteúdo adequado aos ambientes que atuar e; estabelecer uma atuação responsiva.

1)A presença online é o marco inicial de ingresso aos interessados em atingir um público maior e cada vez mais conectado no ambiente web. Além de estar no portal de periódicos de sua instituição e possuir uma página oficial, criar um blog ou mesmo perfis em mídias sociais é uma ação que editores devem considerar para suas revistas.

O Blog de Olho na $C I^{2}$, por exemplo, foi criado no âmbito no âmbito do periódico secundário Revista Pesquisa Brasileira em Ciência da Informação e Biblioteconomia (PBCIB) e tem por objetivo promover maior aproximação entre o público-alvo da revista (FREIRE; SANTOS; NASCIMENTO, 2013).

2)O conteúdo deve ser elaborado de maneira criativa e pensado de forma alinhada ao escopo da política editorial da revista, com definição de linguagem (adequada à mídia social escolhida), frequência e periodicidade. As pessoas tendem a interagir com conteúdos que as interessem, e é comum buscarem algum tipo de 'filiação' com produtores desses conteúdos (assinar o blog, curtir a página no Facebook, seguir no Twitter) e recomendá-los.

Ainda sobre o conteúdo, para reduzir a sensação de acúmulo de mais trabalho para editores e equipe, uma ação que pode ser feita que é o convite para que autores de artigos publicados na revista, escrevam sobre suas pesquisas com uma linguagem mais acessível, com link para acesso ao artigo. O blog SciELO em Perspectiva ${ }^{3}$ é um bom exemplo desse tipo de ação.

A relação dialógica a ser estabelecida entre o conteúdo e os usuários pode ser considerada o maior desafio no marketing digital. Estar online e

\footnotetext{
${ }^{2}$ Blog De Olho Na CI é executado no âmbito da rede de projetos Laboratório de Tecnologias Intelectuais (LTi) da Universidade Federal da Paraíba (UFPB) e possui registro no Internet Blog Serial Number (IBSN) de número 383-6651-52-4. Disponível em: <http://www.deolhonaci.com/>. Acesso em: 23 jul. 2014.

${ }^{3}$ Disponível em: <http://blog.scielo.org/ >. Acesso em: 5 set. 2014. O blog SciELO em Perspectiba tem como objetivo "compartilhar informação e conhecimento orientado para o desenvolvimento da comunicação científica. Com a perspectiva de se constituir um veículo de referência da comunidade relacionada ao SciELO e à comunicação e à avaliação da pesquisa científica, o blog está aberto à colaboração de editores, pesquisadores, profissionais e estudantes de informação e comunicação científica.
} 
com conteúdo constantemente atualizado não é suficiente para o bom desempenho nos ambientes digitais. Uma vez que a web 2.0 vai propiciar possibilidades de interação com o conteúdo, com espaços de participação e colaboração, é necessário manter uma atuação atenta e responsiva.

A dedicação a essas três questões é fundamental para a geração e acompanhamento das principais métricas que o marketing digital tem a oferecer ao marketing científico: visibilidade, influência, engajamento e conversão (MACEDO, 2014) também vistas como indicadores de valores construídos na rede (RECUERO, 2009).

A visibilidade se refere à capacidade de alcance do conteúdo exposto, sendo uma medida relacionada à audiência, tamanho do público atingido, volume e frequência (SOUZA, 2009). De acordo com Macedo (2014, p. 32) entender quem são e quantas são as pessoas alcançadas "é fundamental para que se possa haver efetivamente planejamento e um correto direcionamento de conteúdo". Pode ser medida pelo número de acessos, visualizações, fontes de tráfego, seguidores ou fãs que um blog ou perfil obtém.

Ainda que alcance um grande público é necessário mensurar o quanto tal público pode ser influenciado nas ações de audiência. A influência relaciona-se com a autoridade de quem publica, ou seja, sua reputação. Essa medida tem o objetivo de indicar, por exemplo, o quanto o periódico ou seus artigos, tem influência sobre o público, e mesmo o papel de determinados usuários/influenciadores na audiência e formação de opinião de um grupo. Já existem serviços que se utilizam de algoritmos capazes de gerar pontuação de reputação automática, baseado em comentários e interações dos usuários em relação a um influenciador (SILVA, 2013).

Para além do que o número de usuários, leitores e interessados que se alcança é importante que o conteúdo gerado obtenha o mínimo de entrosamento e interação do público e com o público. $O$ engajamento pode ser visto como uma audiência com qualidade uma vez que indica uma 'reação' do usuário frente ao conteúdo. As medidas de engajamento são os principais indicadores de desempenho seja de sucesso ou falha das campanhas de marketing digital (SILVA, 2013). O número de vezes que um artigo é compartilhado, os comentários e recomendações de leitura que recebe, são exemplos de medidas de engajamento.

Por fim, tem-se as métricas de conversão, com função de medir resultados e eventos relevantes, geralmente são quantitativas e baseadas em metas (MACEDO, 2014). As visitas que o website do periódico ou determinado artigo recebe originadas de pesquisas realizadas no Google podem ser consideradas de conversão de pesquisa. Tais visitas relevam o quanto o conteúdo é atrativo em relação aos demais itens do resultado de busca (VOLMMER; PRECOURT, 2008). O número de downloads dos artigos também pode ser considerado uma medida de conversão, e importante de ser acompanhada.

Um plano de marketing científico digital aplicado ao periódico eletrônico pode considerar essas quatro métricas básicas. Quando a análise se volta para os artigos do periódico e a para a compreensão da 
atenção que esses recebem na rede, por onde circulam e quem os coloca em circulação, adentra-se em um novo campo dos estudos métricos da informação científica na web social, que tem sido chamado de altmtetria.

\section{Altmetria: visibilidade e engajamento}

A altmetria tem sido considerada como métricas alternativas da comunicação científica. Assemelha-se um pouco ao fator de impacto, mas é mais social e responsivo. Os estudos altmétricos medem aspectos desconsiderados nas citações, como, onde um artigo está sendo baixado, lido, compartilhado e discutido, o que amplia o olhar para a visibilidade e - alcance dos resultados de investigação, para além da comunidade científica.

Para Souza (2014, p. 71) a altmetria não se apresenta apenas "como uma evolução técnica dos estudos métricos da informação (bibliometria, informetria, webometria, cientometria etc), agora aplicados à web social; mas também como uma reação política à crise do sistema de comunicação científica". E segundo Thelwall (2014) estas novas métricas poderiam auxiliar estudiosos e acadêmicos a encontrar artigos importantes e talvez também avaliar o impacto de seus próprios artigos.

Existem ferramentas que podem ser utilizadas para acompanhamento, coleta e análise de dados altmétricos. Algumas de uso mais individual no qual o pesquisador analisa seu impacto e a repercussão de sua pesquisa como o ImpactStory ${ }^{4}$ que monitora o impacto de conteúdos disponibilizados online quanto às menções, tweets, citações em blogs, números de downloads e diversas outras formas alternativas de referência (PIWOWAR, 2013; GOUVEIA, 2013).

Outras são voltadas para pesquisa de artigos de periódicos científicos, como é o caso do Altmetric. com $^{5}$, que localiza e avalia quanto a sua citação em blogs, mídias sociais e gestores de referências (Mendeley, CiteULike e o Zotero), qualquer artigo que possua um Digital Object Indentifier - DOI, ou outro identificador padrão (PIWOWAR, 2013; GOUVEIA, 2013) como o Uniform Resource Identifier - URI.

O serviço do Altmetric.com que pode ser adquirida a um custo de $£ 3,000$ (três mil libras) anuais para editores e equipe ${ }^{6}$, tem se consolidado como ferramenta mais utilizada e de maior adesão entre editores e bases como Elsevier/Scopus, Springer e a SciELO, tendo sido analisada por diversas pesquisas (ADIE; ROE, 2013; PIWOWAR, 2013; COSTAS; ZAHEDI; WOUTERS, 2014; ALPERIN, 2014; ARAÚJO, 2014a; NASCIMENTO; ODDONE, 2014).

Para Taylor, Zijlstra e Colledge (2014) a relação da Elsevier com a altmetria remonta ao início deste campo emergente. Em novembro de

\footnotetext{
${ }^{4}$ Disponível em: <http://impactstory.org>. Acesso em: 13 nov. 2014.

${ }^{5}$ Disponível em: <http://altmetric.com>. Acesso em: 11 abr. 2013.

${ }^{6} \mathrm{O}$ valor dá acesso completo à versão de editor do Altmetric Explorer para editores e equipe (até cinco usuários) e permite a importação de suas próprias contagens de downloads, citações e outras estatísticas. Nessa versão é possível realizar consultas com aplicação de filtros por revista, artigos, palavras-chave, editora, ISSN ou prefixo DOI, bem como gerar e salvar relatórios no formato de planilha (.xIs). Informações sobre opções de planos para o uso institucional ou de aplicação customizada da plataforma estão disponíveis em: <http://www.altmetric.com/plansandpricing.php>. Acesso em: 11 abr. 2013.
} 
2011, Elsevier anunciou o projeto vencedor do Apps for Science Challenge, cuja solução media a atenção que os artigos recebiam via mídias sociais e sites de notícias on-line. A pontuação Altmetric.com e sua visualização são exibidas no banco de dados de resumos e citações da Scopus, desde 2012. Elas aparecem na barra lateral da página do documento quando os dados estão disponíveis para o artigo que está sendo visto.

Em novembro de 2013, foram lançados os pilotos que utilizam visualizações semelhantes na plataforma de conteúdo de investigações ScienceDirect, ${ }^{7}$ e nas páginas iniciais dos periódicos da Elsevier. Com base nos resultados e feedback positivo, ScienceDirect e os websites das revistas estão indicar dados altmétricos a um conjunto cada vez mais amplo de revistas (TAYLOR; ZIJLSTRA; COLLEDGE, 2014).

O novo documento Scielo (2014, p. 25) sobre critérios, política e procedimentos para a admissão e a permanência de periódicos científicos, aponta a agregação de um "indicador de influência e presença dos artigos na Web" de "desempenho de cada periódico, medido com base nas referências recebidas por artigo nas redes sociais, é avaliado, sempre que possível, em conjunto com os periódicos da mesma área". O documento afere à Altmetric.com a medição de indicadores da presença na Web e nas redes sociais.

O Altmetric.com divulgou recentemente o ranking ${ }^{8}$ dos 100 artigos com melhor desempenho no ano de 2014, ou seja, a lista dos artigos que mais receberam atenção online em termos de audiência e interações. De acordo com Araújo (2014a, p. 5) "saber se os artigos passam a circular na web social e compreender as métricas que sustentam seu fluxo na rede contribui para desenhar seu quadro altmétrico" e possivelmente verificar, a partir da atenção que recebem se resulta em futuras citações.

A Figura 1 apresenta o quadro altmétrico do artigo de Kramer, Guillory e Hancock (2014) que tem como título "A evidência experimental do contágio emocional em larga escala nas redes sociais", o qual obteve o primeiro lugar no ranking. Publicado no v.111, n.24 do periódico Proceedings of the National Academy of Sciences of the United States of America, após a coleta de todas as mensagens que agregavam o artigo, bem como dos perfis a elas associados, o artigo alcançou uma pontuação de 5.194 .

\footnotetext{
7 Plataforma mantida pela Elsevier para acesso a textos completos de artigos de periódicos científicos e capítulos de livros. Conta com cerca de 2.500 revistas e 26 mil livros. Disponível em: <http://www.sciencedirect.com/>. Acesso em: 17 maio 2013.

8 Altmetric 2014 Top 100. Disponível em: <http://www.altmetric.com/top100/2014/>. Acesso em: 19 nov. 2014.
} 
Figura 3 - Quadro altmétrico do artigo de Kramer, Guillory e Hancock (2014)

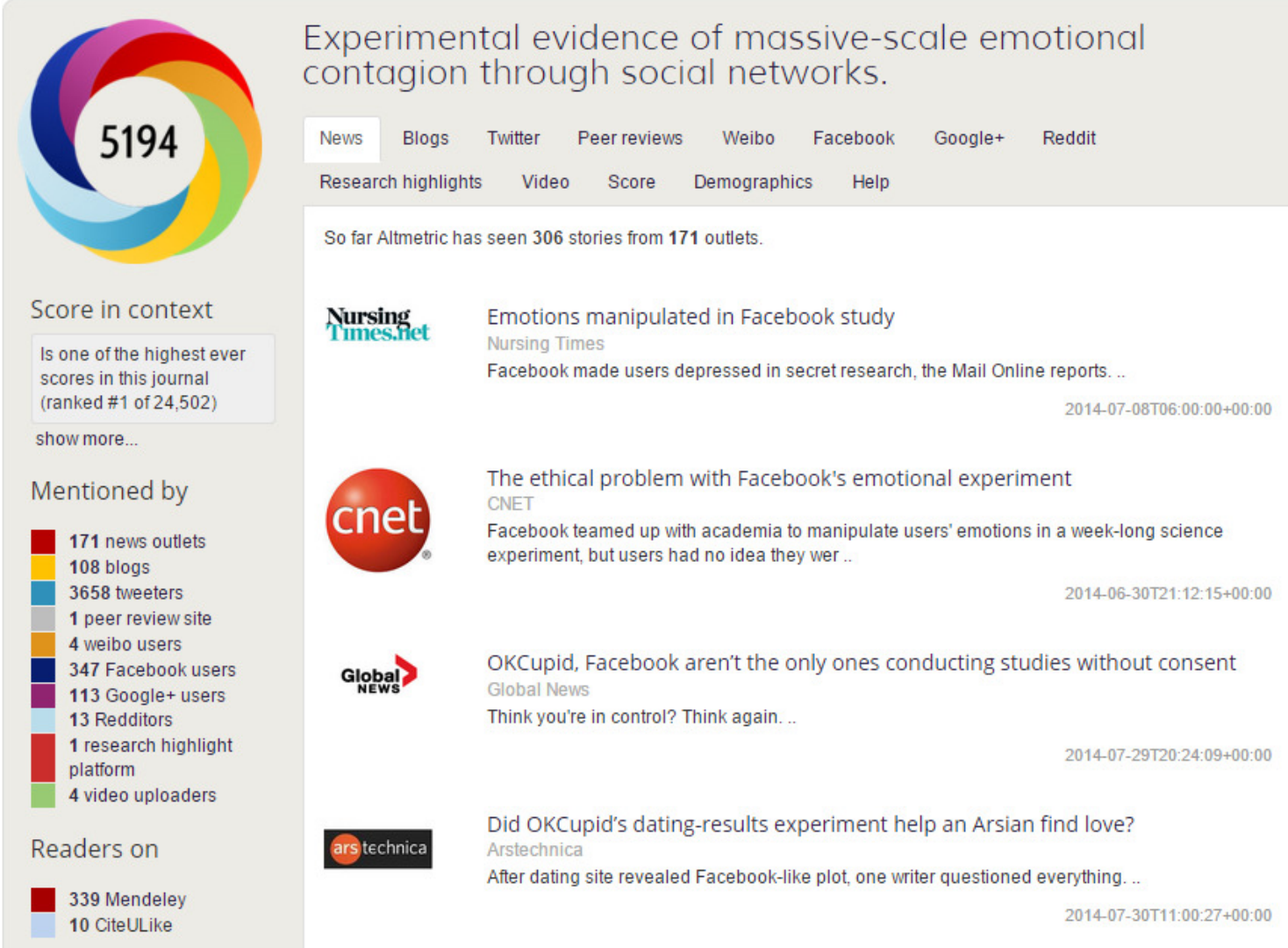

Fonte: ALTMERIC (2014) $)^{9}$.

A pontuação Altmetric é uma medida geral da atenção que um artigo ou conjunto de dados tem recebido online, e deve refletir (i) a visibilidade - quantidade de atenção recebida - em geral, quanto mais pessoas visualizam ou falam sobre um artigo maior sua pontuação; (ii) influência e engajamento - a qualidade da atenção, local postado e reputação de quem posta. Uma matéria num portal de notícia conta mais que a de um post no Facebook. Atenção de um pesquisador conta mais do que a atenção de um Twitter bot automatizado (ALTMETRIC, 2014).

De acordo com a figura o artigo foi mencionado por 3.658 usuários do Twitter e compartilhado por 347 usuários do Facebook e 113 usuários do Google+. Os gerenciadores de referências indicam 349 leitores, sendo 339 do Mendeley e 10 do CiteULike. O artigo chamou a atenção da mídia internacional e foi citado em matérias de 171 portais de notícia, além de ser referenciado em postagens de 108 blogs.

Os dados confirmam a altmetria como um campo promissor para a "compreensão do impacto da pesquisa científica na era digital e das redes sociais; um campo que abre novas perspectivas para uma abordagem multidimensional sobre a visibilidade e o alcance das pesquisas, considerando inclusive uma nova relação entre ciência e sociedade" (FAUSTO, 2013).

\footnotetext{
${ }^{9}$ Disponível em: <http://www.altmetric.com/details.php?citation id=2397894>. Acesso em: 19 nov. 2014.
} 
O que se percebe é que com abordagens altmétricas é possível qualificar a visibilidade do artigo científico e a atenção online que este recebe. Nessa perspectiva as noções de alcance e audiência, bem como as concepções de rede (co-citação e colaboração científica), geralmente pautadas em indicadores quantitativos, como nos estudos tradicionais de citação, se ampliam, pois deixam de se restringir apenas à comunidade científica para atingir um público maior, que munido dos recursos da web social passa a interagir com o conteúdo (no compartilhamento, avaliação e discussão dos artigos).

A altmetria, longe de substituir medidas tradicionais de citação como os fatores de impacto ou o índice $h$, "desponta como um método complementar de mensurar a repercussão da produção científica e de monitorar a forma como artigos científicos se disseminam e são discutidos por pesquisadores e o público leigo imediatamente depois de sua divulgação" (MARQUES, 2014, p. 47).

Ainda sobre a complementaridade que apresenta, ao explorar as possibilidades práticas da altmetria fica evidente que ela mensura impactos distintos aos da análise de citação. Para Priem, Piwowar e Hemminger (2012) métricas alternativas e tradicionais se relacionam, e isoladas, nenhuma delas é capaz de descrever o quadro completo da comunicação científica, mas juntas enriquecem as possibilidades de avaliação (FAUSTO, 2013).

\section{Considerações finais}

O marketing científico digital pode ser considerado um conjunto de ações planejadas e orientadas, com base nas teorias e estratégias do marketing, utilizando-se dos recursos de comunicação interativa da web social, os quais são aplicados, com o intuito de estreitar o relacionamento com e para além da comunidade científica a qual se aplica.

Com os avanços tecnológicos, o advento e evolução da web, a visibilidade tem sido cada vez mais moeda corrente para análises da presença online e dos impactos sociais que ela gera, sobre tudo na ampliação do debate acadêmico e na popularização de textos científicos a públicos não especializados.

Para a geração e acompanhamento da análise de presença na web e indicadores de menções nas mídias sociais, os editores precisam providenciar um número identificador único. Para SciELO (2014, p. 19) "todos os artigos dos periódicos SciELO devem ter o respectivo DOI registrado na base de dados da agência CrossRef. O DOI pode ser obtido pelo SciELO ou pelo periódico". Para o Latindex, de acordo com AlonsoGamboa (2014, p. 47) "todos os links, incluídos na revista devem ser seguros", sendo de um "Uniform Resource Identifier (URI) ou DOI".

A SciELO (2014, p. 20) no item "marketing e divulgação" passa a exigir que os periódicos indexados na base devem contar, a partir de julho de 2015, com um "plano operacional de marketing e divulgação", exigindo-se: "a gestão de uma lista atualizada de pesquisadores 
potenciais, autores e usuários nacionais e internacionais, potenciais leitores, assim como de instituições relacionadas".

A partir de julho de 2015 é esperado também que os periódicos "disseminem as novas pesquisas nas redes sociais mais pertinentes, utilizando o Twitter, o Facebook e/ou outros sistemas" além de "desenvolver mecanismos que fomentem a presença nos sistemas orientados a gestão de informação e comunicação de pesquisadores, como são o Academia.edu, Mendeley, ResearchGate, etc" (SciELO, 2014, p. 20).

A inclusão desses itens propostos pelo documento SciELO (2014) é condizente com o momento atual e com o movimento nacional e internacional rumo as novas métricas. O marketing científico digital e a altmetria podem auxiliar os periódicos atenderem a esses quesitos, seja na promoção e divulgação ou mesmo na análise de desempenho. Resta uma reflexão sobre até que ponto, a indicação de um serviço pago como do Altmetric.com, tendo em vista o custo e os padrões que exige, é viável ou não para as revistas brasileiras.

E em caso de inviabilidade financeira ou de padrão, é necessário pensar em caminhos alternativos para geração desses dados. O estudo de Araújo (2014b) seguiu esse percurso e sugere que os dados altmétricos sejam obtidos por meio do uso de Application Programming Interface (API) das mídias sociais, que parametrizado conseguiu obter indicadores dos artigos para o Facebook e Twitter.

\section{Referências}

ADIE, E.; ROE, W. Altmetric: enriching scholarly content with article-level discussion and metrics. Learned Publishing, v. 26, n. 1, p. 11-17, jan. 2013.

ALONSO GAMBOA, J. O. As características editoriais das revistas Latino Americanas desde a perspectiva de Latindex. In: SEMINÁRIO LATINOAMERICANO SOBRE COMUNICAÇÃO CIENTÍFICA E INDEXADORES, $1 ., 3 \mathrm{e}$ 4 nov. 2014, Rio de Janeiro. Anais... Rio de Janeiro: UERJ, 3/4 nov. 2014. p. 1-75. Disponível em: <https://www.dropbox.com/s/mvvym049yuog6da/20141104 Latindex Al onso Gamboa.pdf >. Acesso em: 3 nov. 2014.

ALPERIN, J. P. Exploring altmetrics in an emerging country context. In: AN ACM WEB SCIENCE CONFERENCE 2014 WORKSHOP, 4., 23 jun. 2014. Bloomington, Indiana. Disponível em: http://dx.doi.org/10.6084/m9.figshare.1041797. Acesso em: 11 jun. 2014.

ALTMETRIC. How is the Altmetric score calculated? 2014. Disponível em: <http://support.altmetric.com/knowledgebase/articles/83337-how-isthe-altmetric-score-calculated->. Acesso em: 11 abr. 2013.

ARAÚJO, R. F. Cientometria 2.0, visibilidade e citação: uma incursão altmétrica em artigos de periódicos da ciência da informação. In: ENCONTRO BRASILEIRO DE BIBLIOMETRIA E CIENTOMETRIA (EBBC), 4., 
Recife, maio 2014. Anais... Recife: UFPE, 2014. p. 1-7. Disponível em: $<$ http://www.brapci.inf.br/ repositorio/2014/05/pdf 7e02bbbf55 001438 7.pdf >. Acesso em: 5 jun. 2014a.

ARAÚJO, R. F. Dos dados altmétricos às citações: uma análise da revista Datagramazero. In: ENCONTRO NACIONAL DE PESQUISA EM CIÊNCIA DA INFORMAÇÃO (ENANCIB), 15., Belo Horizonte, 2014. Anais... Belo Horizonte: Ancib, 2014b. p. 2864-2879.

ARAÚJO, R. F.; OLIVEIRA, M.; SILVA, B. F. M.. Cientometria 2.0 e a presença online de pesquisadores. In: ENCONTRO BRASILEIRO DE BIBLIOMETRIA E CIENTOMETRIA (EBBC), 4., Recife, maio 2014. Anais... Recife: UFPE, 2014. p. 1-4. Disponível em: http://www.brapci.inf.br/ repositorio/2014/05/pdf ec91aec4f3 0014461.pdf> Acesso em: 5 jun. 2014.

ARAÚJO, V. M. R. H.; FREIRE, I. M. A rede Internet como canal de comunicação, na perspectiva da Ciência da Informação. Transinformação, Campinas, v. 8, n. 2, p. 45-55, mai-ago1996. Disponível em: $<$ http://periodicos.puc-

campinas.edu.br/seer/index.php/transinfo/article/viewFile/1610/1582 >. Acesso em: 4 mar. 2014.

BERTIN, P. R. B.; FORTALEZA, J. M.; SUHET, A. R. Paradigma atual da comunicação científica e introdução da revista Pesquisa Agropecuária Brasileira (PAB) no canal eletrônico. Perspect. ciênc. inf., Belo Horizonte, v. 12, n. 3, p. 83-95, dez. 2007. Disponível em: $<$ http://www.scielo.br/pdf/pci/v12n3/a07v12n3.pdf >. Acesso em: 10 nov. 2014.

BIZZOCCHI, A. Marketing científico: o papel do marketing na difusão da ciência. In: CONGRESSO BRASILEIRO DE CIÊNCIAS DA COMUNICAÇÃO, 24., Salvador , 2002. Anais... Salvador: SBEIC, 2002. Disponível em: $<$ http://www.portcom.intercom.org.br/pdfs/d48f2ea87a093314566f93cc5 5e9753d.pdf $>$. Acesso em: 3 abr. 2014.

BOMFÁ, C. R. J. et. al. Marketing científico electrônico: um novo conceito voltado para periódicos electrônicos. Estudos em Comunicação, n. 5,p.193-215, maio 2009.

BOMFÁ, C. R. J. et. al. Marketing aplicado aos periódicos científicos. In: SEMANA DE ENGENHARIA DE PRODUÇÃO SUL-AMERICANA (SEPROSUL), 7., Salto, Uruguai, 2007. Anais... Salto, Uruguai: UDELAR, 2007. p. 1-6. Disponível em: <http://www.kmbusiness.net/images/bomfaseprosul.pdf $>$. Acesso em: 3 jun. 2010.

CARVALHO, A. P. de. A divulgação e o marketing da ciência: uma análise do documentário como instrumento híbrido de comunicação científica pública, por encomenda de instituições públicas de pesquisa à empresa Videociência. 2003. 186f. Tese (Doutorado em Comunicação Social) Universidade Metodista de São Paulo (Umesp), São Paulo, 2003. 
CASTRO, F. de. Impacto nacional. São Paulo: Agência FAPESP: 20 set. 2011. Disponível em: <http://agencia.fapesp.br/impacto nacional/14510/ . Acesso em: 21 out. 2013.

COSTAS, R. ZAHEDI; Z.; WOUTERS, P. Do altmetrics correlate with citations? Extensive comparison of altmetric indicators with citations from a multidisciplinary perspective. Journal of the Association for Information Sciences and Technology. v. 66, n. 10, p. 1401.4321, jan. 2014.

CUENCA, A. M. B. et al. Revistas brasileiras em Saúde Pública: aspectos de edição, forma e conteúdo. In: MUCHERONI, M. L. et al. (Org). Revistas científicas em Ciências da Saúde: visibilidade, forma e conteúdo. São Paulo: Faculdade de Saúde Pública da USP, 2013. p. 71-86.

FAUSTO, S. Altmetrics, altmétricas, altmetrias: novas perspectivas na visibilidade e

no impacto das pesquisas científicas. 2013. Disponível em: $<$ http://blog.scielo.org/blog/2013/08/14/altmetrics-altmetricasaltmetrias-novas-perspectivasna-visibilidade-e-no-impacto-das-pesquisascientificas/\#.U7mOWvldWSp>. Acesso em: 21 jan. 2014.

FERREIRA, S. M. S. P.; TARGINO, M. das G. Mais sobre revistas científicas: em foco a gestão. São Paulo: Senac-SP; Cengage-Learning, 2008.

FERREIRA, A. G.; CAREGNATO, S. E. Visibilidade de revistas científicas: um estudo no Portal de Periódicos Científicos da Universidade Federal do Rio Grande do Sul. TransInformação, Campinas, v. 26, n. 2, p. 177-190, maio/ago. 2014.

FREIRE, I. M.; SANTOS, R. N. R.; NASCIMENTO, B. O. N. Gestão da informação no blog de olho na CI. Inf. Inf., Londrina, v. 19, n. 1, p. 95111, jan./abr. $2014 . \quad$ Disponível em: $<$ http://www.uel.br/revistas/uel/index.php/informacao/article/view/15689 $\angle 14209$ > . Acesso em: 15 out. 2014.

FURNARO, V. M. B. O.; RAMOS, L. M. S. V. C.; CARVALHO, T. As revistas brasileiras de Odontologia e seus canais de divulgação da pesquisa científica. In: MUCHERONI, M. L. et al. (Org). Revistas científicas em Ciências da Saúde: visibilidade, forma e conteúdo. São Paulo: Faculdade de Saúde Pública da USP, 2013. p. 87-112.

GOUVEIA, F. C. Altmetria: métricas de produção científica para além das citações. Liinc em Revista, Rio de Janeiro, v. 9, n. 1, p. 214-227, maio 2013.

\section{INSTITUTO BRASILEIRO DE INFORMAÇÃO EM CIÊNCIA E TECNOLOGIA}

(IBICT). Serviço de Editoração Eletrônica de Revistas: apresenta o sistema de

editoração eletrônica. Disponível em: <http://seer.ibict.br/>. Acesso em: 18 jan. 2013. 
KRAMER, A. D.; GUILLORY, J. E.; HANCOCK, J. T. Experimental evidence of massive-scale emotional contagion through social networks. Proceedings of the National Academy of Sciences of the United States of America, v.111, n. 24, 2014. Disponível em: $<$ http://www.pnas.org/content/111/24/8788.full.pdf+html>. Acesso em: 27 ago. 2014.

MACEDO, T. Métricas de marketing digital e sua aplicação nas ações de marketing das organizações: estudo de caso múltiplos. 2014. $119 f$. Dissertação (Mestrado em Administração) - Universidade Federal do Rio Grande do Sul, Escola de Administração, Programa de Pós-Graduação em Administração, Porto Alegre, 2014.

MARQUES, F. Retuíte ou pereça. Revista Pesquisa FAPESP, n. 221, p. 4647, jul. 2014. Disponível em: <http://revistapesquisa.fapesp.br/wpcontent/uploads/2014/07/pg046-047.pdf>. Acesso em: 14 jul. 2014.

NASCIMENTO, A. G.; ODDONE, N. Uso de indicadores altmétrics na avaliação de periódicos científicos brasileiros em ciência da informação. In: ENCONTRO BRASILEIRO DE BIBLIOMETRIA E CIENTOMETRIA, 4., Recife, maio 2014. Anais... Recife, 2014. p. 1-2. Disponível em:

<http://www.brapci.inf.br/_repositorio/2014/05/pdf_154dd0df78_001431 7.pdf>. Acesso em: 5 jun. 2014.

OLIVEIRA, É. B. P. M. de; NORONHA, D. P. A comunicação científica e o meio digital. Inf. \& Soc.:Est., v. 15, n. 1, p. 5-92, jan./jun. 2005. Disponível em: <http://www.biblionline.ufpb.br/ojs2/index.php/ies/article/view/53/1523> . Acesso em: 12 jun. 2013.

OLIVEIRA, L. A. Estratégias digitais de marketing orientadas à performance das instituições. 2011. 51f. Monografia (Bacharelado em Administração) - Universidade de Brasília, Departamento de Administração, 2011.

PACKER, A. L. A visibilidade dos periódicos do Brasil: SciELO em perspectiva. Disponível em: <http://blog.scielo.org/blog/2014/11/05/avisibilidade-dos-periodicos-do-brasil/ >. Acesso em: 5 nov. 2014.

PACKER, A. L.; MENEGHINI, R. Visibilidade da produção científica. In: POBLACION, D. A.; WITTER, G. P.; SILVA, J.F.M. (Org.). Comunicação e produção científica: contexto, indicadores e avaliação. São Paulo: Angellara, 2006. p. 237-259.

PIWOWAR, H. Introduction altmetrics: what, why and where? Bulletin of the Association for Information Science and Technology, v. 39, n. 4, p.89, Apr./May 2013.

POTTS, K. Web design and marketing solutions for business websites. New York: Friendsoft, 2007. 
PRIEM, J.; PIWOWAR, H. A.; HEMMINGER, B. Altmetrics in the wild: using social media to explore scholarly impact. CoRR, v. 12, n. 3, p. 1-17, mar. 2012.

REBIUN. Science 2.0: the use of social networking in research. Revised and updated. Madrid: REBIUN, 2011. Disponível em: <http://www.rebiun.org>. Acesso em: 15 dez. 2013.

RECUERO, R. Redes sociais na Internet. Porto Alegre: Sulina, 2009.

SANTOS, E.; FREIRE, J.; SILVA, C. Information sharing in science 2.0: challenges and opportunities. In: CHI WORKSHOP - THE CHANGING FACE OF DIGITAL SCIENCE: NEW PRACTICES IN SCIENTIFIC COLLABORATIONS, 1., Boston, abr. 2009. p. 1-2. Disponível em: $<$ www.isi.edu/ qil/diw2012/statements/aragon.pdf $>$. Acesso em: 20 dez. 2013.

SCIENTIFIC ELETRONIC LIBRARY ONLINE (SciELO). Critérios, política e procedimentos para a admissão e a permanência de periódicos científicos na Coleção SciELO Brasil. São Paulo: SciElO, 2014. Disponível em: $<$ http://www.scielo.br/avaliacao/20141003NovosCriterios SciELO Brasil.p df $>$. Acesso em: 23 out. 2014.

SILVA, T. Métricas em mídias sociais. Disponível em: <http://pt.slideshare.net/tarushijio/techday-ufma-metricas-em-midiassociais >. Acesso em: 9 jul. 2014.

SOUZA, I. V. P. Altmetria: métricas alternativas do impacto da comunicação científica. Instituto de Arte e Comunicação Social. Programa de Pós-Graduação em Ciência da Informação. 2014. 105f. Dissertação (Mestrado em Ciência da Informação) - Universidade Federal Fluminense, 2014.

SOUZA, E. Métricas em mídias sociais. Pólvora comunicação, 2009. Disponível em: <http://pt.slideshare.net/interney/mtricas-em-mdiassociais $>$. Acesso em: 18 jan. 2013.

TAYLOR, M.; ZIJLSTRA, H.; COLLEDGE, L. Elsevier is expanding its use of altmetrics. Elsevier Impact Metrics, 14 set. 2014. Disponível em: <http://www.elsevier.com/authors-update/story/impact-metrics/elsevieris-expanding-its-use-of-altmetrics>. Acesso em: 18 jan. 2015.

THEWALL, M. A brief history of altmetrics. Research Trends, n. 37, p. 3-5, jun. 2014. Disponível em: <http://www.researchtrends.com/wpcontent/uploads/2014/05/Research Trends Issue 37.pdf>. Acesso em: 19 jul. 2014.

TORRES, C. A bíblia do marketing digital: tudo o que você queria saber sobre marketing e publicidade na internet e não tinha a quem perguntar. São Paulo: Novatec, 2009.

VILLAMÓN, M. et al. Las Revistas Españolas de Ciencias del Deporte

Incluidas en el Catálogo Latindex. Movimento, Porto Alegre, v. 15, n. 03, p. 13-34, 
junho/setembro de $2009 . \quad$ Disponível em: <http://seer.ufrgs.br/Movimento/article/download/8567/5577>. Acesso em: 09 jun. 2013.

VOLMMER, C.; PRECOURT, G. Always on: adversiting, marketing and media in an era of consumer control. New York: McGraw-Hill Education, 2008.

WALDROP, M. M. Science 2.0: is open access science the future? Scientific American, v. 298, n. 5, p. 69-73, maio 2008. Disponível em: $<$ http://www.nature.com/scientificamerican/journal/v298/n5/pdf/scientific american0508-68.pdf>. Acesso em: 21 set. 2012.

ZIMBA, H. F.; MUELLER, S. P. M. Colaboração internacional e visibilidade científica de países em desenvolvimento: o caso da pesquisa na área de medicina veterinária em Moçambique. Inf. \& Soc.:Est., João Pessoa, v. 14, n. $1, \quad$ p. 45-68, 2004. Disponível em: <http://www.ies.ufpb.br/ojs2/index.php/ies/article/view/71/1544>. Acesso em: 11 jun. 2014. 